\title{
Specific-heat anomaly of a ferromagnet in finite magnetic fields
}

\author{
E. Scheer, H. Claus, ${ }^{*}$ J. Wosnitza, and H. v. Löhneysen \\ Physikalisches Institut der Universität Karlsruhe, Engesserstrasse 7, D-7500 Karlsruhe 1, Federal Republic of Germany
}

(Received 3 April 1989)

\begin{abstract}
Low-temperature specific-heat data of two ferromagnetic $\mathrm{Eu}_{x} \mathrm{Sr}_{1-x} \mathrm{~S}$ samples $(x=1$ and 0.7$)$ in external magnetic fields between 0 and $50 \mathrm{mT}$ are reported. It is observed that the sharp zerofield anomaly persists in finite fields with the peak temperature shifting towards lower temperature with increasing field. This apparent decrease of the Curie temperature, previously found in other systems, is explained in terms of demagnetization effects alone, the field dependence of the peak temperature being determined by $\beta$, the critical exponent of the spontaneous magnetization.
\end{abstract}

Strictly speaking, any paramagnetic-to-ferromagnetic phase transition is suppressed in finite magnetic fields. This is due to the fact that in finite fields a magnetization exists in the sample at all temperatures and a critical temperature, which in zero field signals a symmetry breaking, no longer exists. Thus, in magnetization measurements great care has to be taken to extrapolate to low enough magnetic fields in order to obtain the true critical behavior. Over many years considerable effort has been put into understanding the analytical form of this extrapolation (critical isotherms).

Much less is known of how the specific-heat anomaly associated with a ferromagnetic transition is modified in finite magnetic fields. ${ }^{1,2}$ In a recent comprehensive study of the critical behavior (in zero field) of the random ferromagnet $\mathrm{Eu}_{x} \mathrm{Sr}_{1-x} \mathrm{~S}$, we have investigated the specificheat anomaly occurring in these compounds at their Curie temperature. $^{3}$

In this paper we present new data which show that a fairly sharp specific-heat anomaly persists in finite magnetic fields with the peak of the anomaly occurring at progressively lower temperatures as the magnetic field is increased. We demonstrate quantitatively that this apparent decrease of $T_{c}$ is a consequence of demagnetization effects alone and not due to the internal critical behavior of these materials, as has been suggested some time ago. ${ }^{1}$ These results are consistent with recent measurements on the $\mathrm{LiHoF}_{4}$ ferromagnet. ${ }^{2}$

Specific-heat measurements were performed on a EuS and $\mathrm{Eu}_{0.7} \mathrm{Sr}_{0.3} \mathrm{~S}$ single crystal. The crystals were grown at about $2500^{\circ} \mathrm{C}$ in a closed tungsten crucible. ${ }^{4}$ The approximate dimensions were $1 \times 1 \times 3 \mathrm{~mm}^{3}$ with the EuS crystal being rather irregular in shape. The specific heat was determined by a standard heat-pulse method with the crystals being mounted on a sapphire sample holder. ${ }^{5}$ The temperature resolution was better than $1 \mathrm{mK}$, the absolute accuracy of the specific heat being about $1 \%$.

Figure 1 displays low-temperature specific-heat data for two $\mathrm{Eu}_{x} \mathrm{Sr}_{1-x} \mathrm{~S}$ samples versus temperature $T$. For both samples the temperature of the zero-field cusp agrees well with the independently determined Curie temperature: ${ }^{6}$ $T_{C}=16.46$ and $8.48 \mathrm{~K}$ for the $x=1.0$ and 0.7 samples, respectively.

One would expect that in finite magnetic fields the magnetization is stabilized by the field and the breakup of the magnetic correlation should occur at higher temperatures. Indeed, the specific-heat curves $C_{H}(T)$ in a magnetic field $H$ cross each other within a narrow $T$ range, with $C_{H}(T)$ being largest for the highest field at higher temperatures. However, the sharp zero-field cusps persist in finite field with only a small degree of broadening, the cusp tempera-

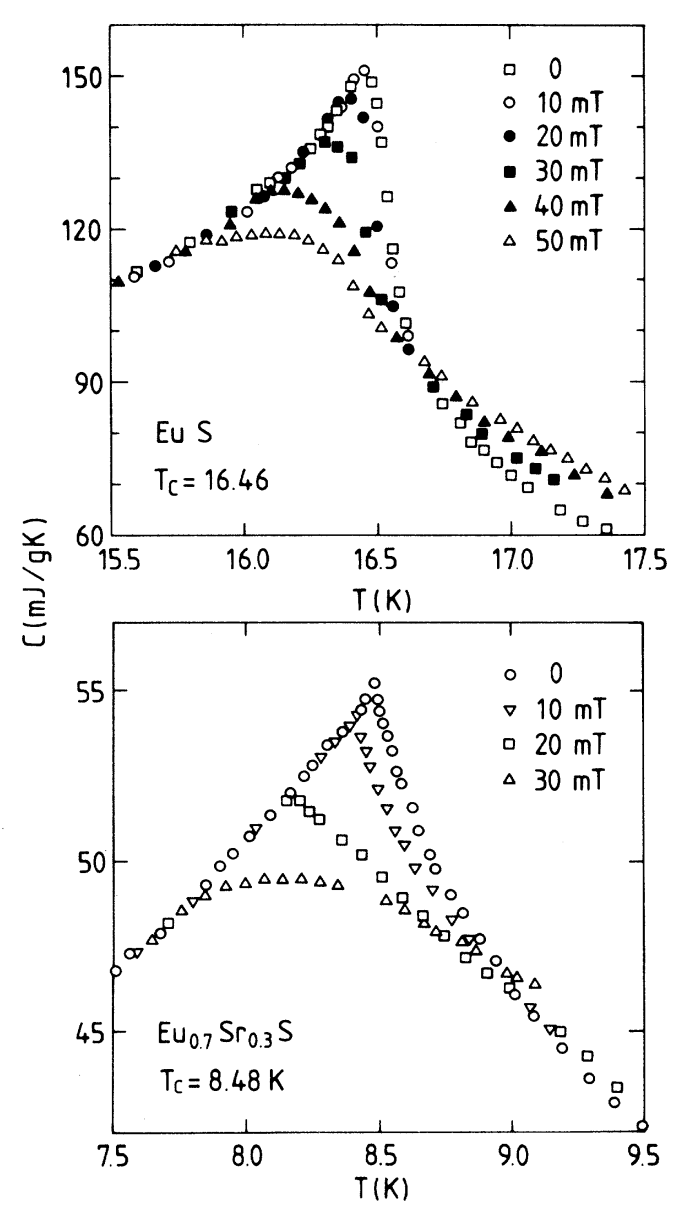

FIG. 1. Specific heat $C_{H}(T)$ in various external magnetic fields $0 \leq H \leq 50 \mathrm{mT}$ vs temperature $T$ for two $\mathrm{Eu}_{x} \mathrm{Sr}_{1-x} \mathrm{~S}$ samples with $x=1.0$ and 0.7 , and with Curie temperatures of $T_{C}=16.46$ and $8.48 \mathrm{~K}$, respectively. 
tures being systematically lowered with increasing field. At first sight this might be interpreted as a field-induced decrease of the ordering temperature. In the following, we will show quantitatively that this suppression of the specific-heat cusps is, rather, a direct consequence of demagnetization effects and is therefore not representative of the intrinsic thermodynamic behavior of the samples.

As can be seen in Fig. 1, at low enough temperatures the specific heat $C_{H}(T)$ in a magnetic field $H$ is independent of $H$ and equal to $C_{0}(T)$, the specific heat in zero field. With increasing temperatures, $C_{H}(T)$ starts to deviate from $C_{0}(T)$ rather suddenly at a temperature $T_{m}$, which depends on the applied magnetic field. This behavior is quite similar to that recently observed for the Ising ferromagnet $\mathrm{LiHoF}_{4}{ }^{2}$ The independence of $C_{H}(T)$ of $H$ at lower temperatures is a trivial consequence of the fact that the sample shields the applied field $H$, causing the internal field $H_{i}=H-N M$ to be zero ( $N$ is the demagnetization factor, $M$ is the magnetization). The sample accomplishes this by adjusting its domain structure to produce a net magnetization of $M=(1 / N) H \cdot{ }^{1,2,7}$ In order for the sample to be able to completely shield $H$, it has to be ideally soft with no hysteretic behavior. This is indeed true for the $\mathrm{Eu}_{x} \mathrm{Sr}_{1-x} \mathrm{~S}$ samples. ${ }^{6}$ Because $H_{i}$ is the relevant field responsible for the intrinsic thermodynamic behavior of the sample, it is of no surprise that as long as $H_{i}=0, C_{H}(T)$ is independent of $H$, the applied magnetic field. ${ }^{1,2}$

With increasing temperatures, the magnetization of the individual domains, i.e., the spontaneous magnetization $M_{S}$, decreases according to $M_{S}=M_{0}\left(1-T / T_{C}\right)^{\beta}$, where $M_{0}$ is the spontaneous magnetization at zero temperature, $T_{C}$ the Curie temperature, and $\beta$ the critical exponent of $M_{S} .{ }^{6}$ Because of this decrease of $M_{S}$ for increasing $T$, the sample has to align more and more domains to keep its magnetization at $M=(1 / N) H$, the value necessary for $H_{i}=0$. With increasing temperature, a point $T^{*}$ will be reached at which all domain walls are driven out of the sample and $M=M_{S}$. This is the maximum magnetization possible for the sample and, at $T^{*}, M_{S}$ is just enough to shield the external field. At any higher temperatures $\left(T>T^{*}\right)$, the magnetization of the sample, now always being $M_{S}$, is too small to shield the external field and the internal field $H_{i}=H-N M$ is finite. Thus,

$$
H_{i}=H-N M_{0}\left(1-T / T_{C}\right)^{\beta} \text { for } T^{*} \leq T \leq T_{C},
$$

and

$$
H_{i}=0 \text { for } T \leq T^{*} .
$$

The larger the magnetic field $H$ the lower $T^{*}$ is. In the above consideration, we neglected for simplicity the fieldinduced magnetization, i.e., the magnetization induced by a finite internal field, in addition to the spontaneous magnetization: $M=M_{S}+\chi(T) H_{i}$ for $T^{*} \leq T \leq T_{C}$.

We now show that the temperature $T_{m}$, at which $C_{H}(T)$ starts deviating from $C_{0}(T)$ (see Fig. 1), is just the temperature $T^{*}$, the temperature above which $H_{i}$ is no longer zero. The sudden decrease in $C_{H}(T)$ for $T>T_{m}$ is then due to the rather fast increase in $H_{i}$ with increasing temperature [Eq. (1)]. The closer $T^{*}$ is to $T_{C}$, the faster $H_{i}$ increases, i.e., the faster the specific heat is suppressed. The somewhat larger rounding observed for the $x=1$ crystal is probably due to the rather irregular shape of this sample leading to a nonuniform internal field.

Using Eq. (1) with $T^{*}=T_{m}$, we can now predict a field dependence of the temperature $T_{m}$ of the specific-heat cusp. Setting $H_{i}=0$, Eq. (1) yields an equation for $T_{m}$ :

$$
0=H-N M_{0}\left(1-T_{m} / T_{C}\right)^{\beta}
$$

or

$$
\log _{10}\left(\Delta T / T_{C}\right)=\beta^{-1} \log _{10}\left(H / N M_{0}\right),
$$

where $\Delta T=T_{C}-T_{m}$ is the decrease of the cusp temperature. Figure 2 displays, on a $\log -\log$ plot, $\Delta T$ vs $H$ for both samples investigated. The error bars correspond to the precision with which $T_{m}$ can be determined from the data in Fig. 1. The absolute value of $\Delta T$ depends on both $N$ and $M_{0}$, which are not well known for our samples. Thus, the absolute values of $\Delta T$ in Fig. 2 are of no significance. However, in the log-log plot of Fig. 2, the data should lie on a straight line with slope $\beta^{-1}$. Also shown in Fig. 2 are results for the Ising ferromagnet LiHoF $_{4}$ (Ref. 2) with $H$ scaled down by a factor of 10. The lines through the points in Fig. 2 have been drawn with a slope of $\beta^{-1}$ with $\beta=0.365$ and 0.425 , the critical exponents of the spontaneous magnetization for EuS and $\mathrm{Eu}_{0.7} \mathrm{Sr}_{0.3} \mathrm{~S}$ as taken from the literature, ${ }^{8,9}$ and $\beta=0.325$ for the Ising ferromagnet $\mathrm{LiHoF}_{4}{ }^{8}$ As can be seen in Fig. 2 , these slopes are in excellent agreement with the experi-

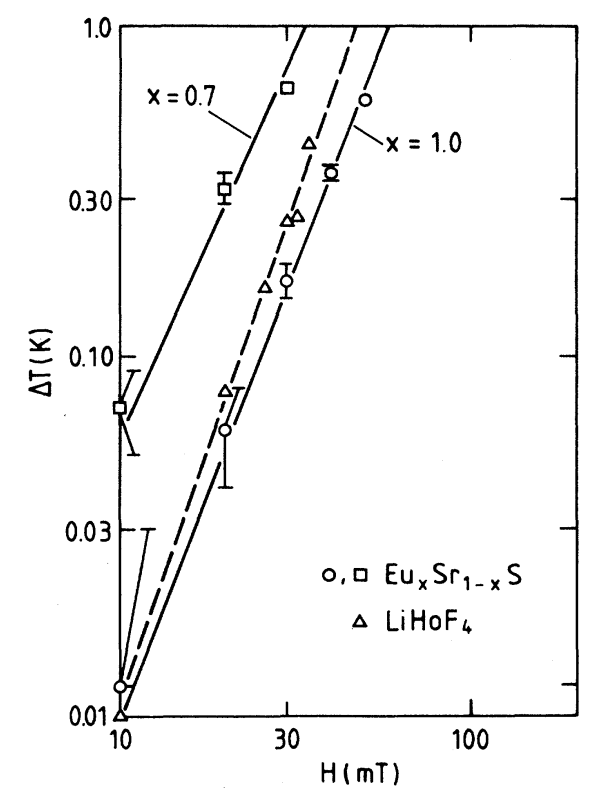

FIG. 2. Decrease of the specific-heat cusps $\Delta T=T_{C}-T_{m}$ vs external field $H$ on a $\log -\log$ scale. The lines through the $\mathrm{Eu}_{x} \mathrm{Sr}_{1-x} \mathrm{~S}$ data points are drawn with slope of $\beta^{-1}$ where $\beta=0.365$ and 0.475 for the two samples with $x=1.0$ and 0.7 , respectively. The $\mathrm{LiHoF}_{4}$ data are from Ref. 2 . The actual magnetic fields from those measurements are scaled down by a factor of 10 . The dashed line through these points correspond to the Ising value of $\beta=0.325$. 
mental points. This convincingly proves our identification of the temperature of the specific-heat maximum with $T^{*}$.

In conclusion, we have shown that the specific heat of ferromagnets in a small external magnetic field displays a fairly sharp cusp similar to that observed in zero field. The temperature at which the cusp occurs is systematically lowered with increasing field. We have demonstrated that the cusps in a field are mainly due to the demagnetization effect, causing the internal field to increase suddenly from zero to a finite value as the temperature is raised beyond a certain value. This sudden increase in the internal field causes the observed drop in the specific heat.

In order to calculate this decrease in $C_{H}(T)$, one would have to know the specific heat at constant internal field which can only be determined in the limit of the applied magnetic field $H \gg N M_{S}$, i.e., at either high magnetic fields and/or small demagnetization factors.

One of us (H.C.) acknowledges the financial support of the Deutsche Forschungsgemeinschaft.
${ }^{*}$ Permanent address: Department of Physics, University of Illinois at Chicago, Chicago, IL, 60680

${ }^{1}$ R. B. Griffiths, J. Appl. Phys. 40, 1542 (1969).

${ }^{2}$ G. Mennenga, L. J. de Jongh, and W. J. Huiskamp, J. Magn. Magn. Mater. 44, 59 (1984), and references therein.

${ }^{3}$ J. Wosnitza and H. v. Löhneysen (unpublished).

${ }^{4}$ The crystals were grown by $K$. Fischer, Institut für Festkörperforschung, Kernforschungsanlage Jülich $\mathrm{GmbH}$, Federal Republic of Germany.

${ }^{5}$ K. Albert, H. v. Löhneysen, W. Sander, and H. J. Schink, Cryogenics 22, 417 (1982).
${ }^{6}$ H. Maletta, G. Aeppli, and S. M. Shapiro, J. Magn. Magn. Mater. 31-34, 1367 (1983).

${ }^{7}$ D. W. Carnegie, C. J. Tranchita, and H. Claus, J. Appl. Phys. 50, 7318 (1979).

${ }^{8}$ J. C. LeGuillou and J. Zinn-Justin, Phys. Rev. B 21, 3976 (1980).

${ }^{9} \beta$ for $\mathrm{Eu}_{0.7} \mathrm{Sr}_{0.3} \mathrm{~S}$ was obtained from $\alpha$ (Ref. 3) and $\gamma$ [K. Westerholt, H. Bach, and R. Römer, J. Magn. Magn. Mater. 45, 252 (1984)] via the Rushbrooke relation $\beta=1$ $-0.5(\gamma+\alpha)$. 